\title{
A Disease Around the Corner
}

The Middle Eastern respiratory syndrome coronavirus outbreak in the Republic of Korea has taken the world by surprise for its scale because the Republic of Korea is regarded to have one of the most advanced medical and public health systems worldwide. Now, a question has arisen - what would be the next infectious disease that could affect Korea? Considering the impact of climate change and vector existence in Korea, dengue fever (DF) is the most probable disease to affect Korea in a different aspect.

DF is a mosquito-borne febrile disease caused by dengue virus (DENV) [1]. Four distinct serotypes of the virus (DENV-1-DENV-4) cause various forms of illness, from mild fever to severe dengue [2]. A World Health Organization report shows that an estimated 50 to 100 million annual dengue infections have occurred in more than 100 countries [3].

The virus is transmitted mainly by two mosquito species, Aedes aegypti and Aedes albopictus [2,3]. A. aegypti, the principal vector, lives in regions where the average winter temperature is above $10^{\circ} \mathrm{C}$ [3].

Because both Korea and Japan are located above this winter temperate zone, A. aegypti cannot survive. However, A. albopictus, a secondary vector, is abundant in both countries $[4,5]$. Thus, both countries could be at risk of dengue transmission. In Korea, no indigenous dengue cases have been confirmed up to 2015, and all reported cases were diagnosed among travelers returning from endemic or epidemic countries [6-8]. In Korea, DF has been classified as notifiable infectious disease since August 2000. DF is also a notifiable infectious disease in Japan, designated by the Infectious Disease Control Law in 1999 [9,10]. In Japan, there were DF outbreaks between 1942 and 1945; however, no domestic cases were reported prior to 2014 [9-11] when Japan experienced an unexpected small dengue outbreak in 2014 [12].

In the current issue of Osong Public Health and Research Perspectives, a study aimed to identify the possible outbreak of DF in Korea by comparison of characteristics of dengue cases with those of Japan. In this study, the authors conducted a comparative observation of the epidemiological characteristics and risk factors of DF between Korea and Japan during 2006 to 2010. To compare the epidemiological characteristics of dengue cases imported by travelers or immigration in both Korea and Japan, the authors concentrated on dengue incidence and related risk factors. During 2006-2010, 367 and 589 imported dengue cases were reported in Korea and Japan, respectively. In Korea, the presumptive origins for the dengue infections were Southeast Asia (82.6\%), Southern Asia (13.9\%), Eastern Asia (1.1\%), South America $(0.3 \%)$, Central America (0.3\%), Africa ( $0.3 \%)$, and other countries (1.6\%). In Japan, the origins of the infections were Southeast Asia (69.8\%), Southern Asia (20.0\%), Eastern Asia (1.7\%), South America (2.5\%), Central America (1.2\%), Africa (1.2\%), Oceania (2.4\%), and other countries $(1.2 \%)$. In both countries, more dengue cases were reported for men than for women $(p<0.01)$, and those aged 20 to 30 years accounted for more than $60 \%$ of the total number of cases. The frequency of imported cases in summer and autumn (approximately 70\% of total cases) was similar in both countries because of vacation season [13].

The authors show that there is a similar pattern of imported dengue cases in Korea and Japan. Accordingly, it leads to a conclusion that there is a risk of an autochthonous dengue outbreak in Korea in the near future, similar to the outbreak that occurred in Japan in 2014.

The authors present an important perspective on an emerging infectious disease in Korea. We expect a further study with mathematical model once an index case is introduced in the Korean population and transmits the disease to neighbors, so that it can lead to an estimated number of casualties of the outbreak, and thus provide invaluable information to public health policymakers. 


\section{References}

1. Knipe DM, Howley DM. Fields virology. In: Flaviviridae. 5th ed. Philadelphia (US): Lippincott-Raven Publishers; 2007. Chapter 33; p. 1101-13.

2. World Health Organization. Dengue and severe dengue. Available from: http://www.who.int/mediacentre/ factsheets/fs117/en/ [accessed 28.12.15].

3. World Health Organization (WHO). Dengue: guidelines for diagnosis, treatment, prevention and control. Geneva (Switzerland): WHO; 2009. Chapter 1, p. 3-21.

4. Nihei N, Komagata O, Mochizuki K, et al. Geospatial analysis of invasion of the Asian tiger mosquito Aedes albopictus: competition with Aedes japonicus japonicus in its northern limit area in Japan. Geospat Health 2014 May; $8(2): 417-27$.

5. Takhampunya R, Kim HC, Tippayachai B, et al. Distribution and mosquito hosts of Chaoyang virus, a newly reported flavivirus from the Republic of Korea, 2008-2011. J Med Entomol 2014 Mar;51(2):464-74.

6. Jeong YE, Kim YH, Cho JE, et al. Identification of Dengue Type 1 Virus (DENV-1) in Koreans traveling abroad. Osong Public Health Res Perspect 2011 Jun;2(1):34-40.

7. Park SH, Lee MJ, Baek JH, et al. Epidemiological aspects of exotic malaria and dengue fever in travelers in Korea. J Clin Med Res 2011 May;3(3):139-42.

8. Park JH, Lee DW. Dengue fever in South Korea, 2006-2010. Emerg Infect Dis 2012 Sep;18(9):1525-7.

9. Nakamura N, Arima Y, Shimada T, et al. Incidence of dengue virus infection among Japanese travellers, 2006 to 2010. Western Pac Surveill Response J 2012 Jun;3(2):39-45.

10. Takasaki T. Imported dengue fever/dengue hemorrhagic fever cases in Japan. Trop Med Health 2011 Dec;39(4 Suppl.):13-5.
11. National Institute of Infectious Diseases and Tuberculosis and Infectious Diseases Control Division. Ministry of Health, Labor and Welfare of Japan. Imported dengue fever and dengue hemorrhagic fever in Japan, as of July 2007. IASR 2007 Jul;28:213-4.

12. Kutsuna S, Kato Y, Moi ML, et al. Autochthonous dengue fever, Tokyo, Japan, 2014. Emerg Infect Dis 2015 Mar;21(3): 517-20.

13. Jeong YE, Lee W-C, Cho JE, et al. Comparison of the epidemiological aspects of imported dengue cases between Korea and Japan, 2006-2010. Osong Public Health Res Perspect 2016 Feb;7(1):71-4.

Hae-Wol Cho*

Osong Public Health and Research Perspectives, Korea Centers for Disease Control and Prevention, Cheongju, Korea

College of Medicine, Eulji University, Daejeon,

*Corresponding author

E-mail: hwcho@eulji.ac.kr

Chaeshin Chu**

Osong Public Health and Research Perspectives, Korea Centers for Disease Control and Prevention, Cheongju, Korea

${ }^{* *}$ Corresponding author E-mail: cchu@cdc.go.kr 